\title{
Early work on the role of mitochondria in apoptosis, an interview with Guido Kroemer
}

\author{
G Kroemer $^{\star, 1}$ \\ ${ }^{1}$ CNRS-UMR 8125, Institut Gustave Roussy, Pavillon de Recherche 1, 39 rue Camille-Desmoulins, F-94805 Villejuif, France \\ * Corresponding author: G Kroemer, Tel: + 33-1-42-11-60-46; Fax: +33-1-42-11-60-47; E-mail: kroemer@igr.fr
}

Cell Death and Differentiation (2004) 11, S33-S36. doi:10.1038/sj.cdd.4401448

Published online 14 May 2004

This interview is part of a series of articles to mark the 10th anniversary of Cell Death and Differentiation.

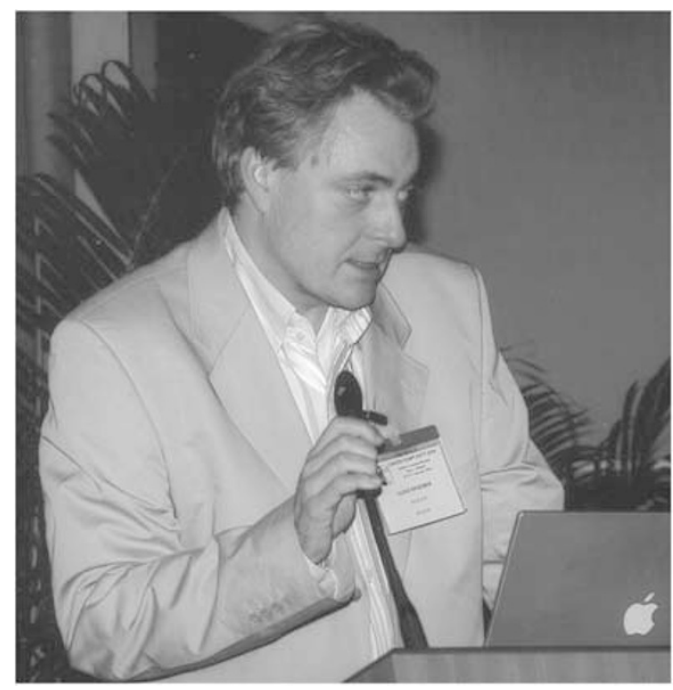

Guido Kroemer currently serves as a Research Director at the French Medical Research Council (INSERM), affiliated to the French National Research Council (CNRS), at the CNRS Research Unit UMR8125 in Villejuif, near to Paris, France. Prior to joining the INSERM/CNRS (1993), Dr. Kroemer was Senior Scientist of the European Community at the Spanish National Research Council (CSIC), at the National Center of Molecular Biology (1990-1992) and at the National Center of Biotechnology (1993). Dr. Kroemer did his post-doctoral training in the Collège de France, Nogent-sur-Marne (1988-1989) and at the University of Innsbruck, Austria, after receiving his Ph.D/M.D. degree at the same University in 1985. He also holds a Ph.D. degree in Biology (Autonomous University of Madrid, 1992). Kroemer's team discovered in 1994 that mitochondrial membrane permeabilization is a critical step in programmed cell death (apoptosis). The group is actually working on the molecular mechanisms of this permeabilization step and its control by oncoproteins from the Bcl-2 family. Furthermore, Kroemer is establishing the mechanistic links between mitochondria and the activation of catabolic enzymes involved in the death process. Particular efforts are undertaken to apply this fundamental knowledge to two pathologies linked to a deregulated apoptosis, namely cancer and AIDS. Kroemer has published over 250 papers in international scientific journals. He is currently the most cited scientist, within the specific are of cell death research, according to the HistCite survey of the University of Pennsylvania, USA (http://www.garfield.library.upenn.edu/histcomp/celldeathdiff_citing_2/hist-aus-lcs.html). Dr. Kroemer is an elected member of the European Molecular Biology organization, serves at the Editorial Board of 12 scientific journals, and has received numerous distinctions, both at the national and at the European levels.
In the mid-1990s the mitochondrion was found to be part of the central control of the apoptotic process affecting mammalian cells. This discovery has had far-reaching consequences for the comprehension of a variety of human diseases linked to an enhanced apoptotic turnover, such as neurodegeneration, stroke, heart ischemia and AIDS. Moreover, it has become clear that the relative apoptosis resistance of cancer cells may be explained by an alteration of the mitochondrial cell death control. According to the Medline database, more than 7400 papers have now been published on mitochondria and apoptosis. Here, Cell Death and Differentiation asks Guido Kroemer about the early work on mitochondria.

\section{CDD: When did you first hear about apoptosis?}

Late, when I was beginning to work on peripheral T-cell tolerance in Madrid, in 1989. One mechanism through which autoreactive $\mathrm{T}$ lymphocytes are hindered from attacking the immunological self-resides in their physical elimination or 'deletion', and I came across the 'a word' when I read the paper by Wyllie ${ }^{1}$ on the oligonucleosomal 'ladder-type' DNA fragmentation of glucocorticoid-treated thymocytes. At that time, back in the 1980s, there was a broad consensus that apoptotic cell death was a nuclear process involving the activation of endonucleases. Chromatin condensation and chromatinolysis were considered as pathognomonic and responsible for apoptosis. So, we treated mice with Staphylococcus aureus enterotoxin B (SEB), a superantigen whose injection can induce T-cell tolerance through activationinduced cell death. ${ }^{2}$ We then recovered splenic $T$ cells and investigated whether they had become apoptotic in vivo, after SEB injection. Neither SEB-treated peripheral $T$ cells nor glucocorticoid-treated splenic $T$ cells (that we considered as our internal positive control) did show any sign of chromatin condensation or DNA fragmentation, when analyzed immediately after their isolation from the animal. Only thymocytes did show such changes ex vivo. However, when we cultured 
isolated splenocytes for $1 \mathrm{~h}$ in vitro, we did find chromatin condensation and DNA fragmentation, and this phenomenon was only observed when the animals had been pretreated with SEB or glucocorticoids. ${ }^{2,3}$ These experiments led us to the frustrating conclusion that apoptosis of peripheral T cells was an in vitro artefact, which only could be observed once the lymphoid tissue had been dissociated.

\section{CDD: How was the role of mitochondria first identified?}

It was exactly this discrepancy between the massive disappearance of peripheral $\mathrm{T}$ cells (in some experimental conditions around $10 \% / \mathrm{h}$ ) and the absence of apoptotic markers such as DNA fragmentation, which prompted us to search for alternative markers of imminent cell death. We reasoned that dying peripheral $\mathrm{T}$ cells were probably eliminated by the phagocytic system before they manifested nuclear apoptosis. So, once I had moved to a laboratory in Villejuif, in the outskirts of Paris, at the end of 1993, I decided to look for biochemical alterations that would distinguish normal T cells and 'preapoptotic' T lymphocytes. We defined 'preapoptotis' as a state at which cells with a normal morphological appearance would be condemned to die. As an immunologist, I attempted to identify fluorochromes that label preapoptotic cells, hoping that such dyes would be useful for the cytofluorometric identification and purification of the dying subpopulation. We looked at a series of markers designed to measure alterations in cellular biochemistry such as shifts in $\mathrm{pH}$ or cation concentrations, changes in membrane permeability, lysosomal change and so on. Finally, we discovered that a subpopulation of those $\mathrm{T}$ cells that are eliminated by the injection of SEB exhibited a reduced incorporation of $\mathrm{DiOC}_{6}{ }^{3}$ a dye that indirectly measures the mitochondrial transmembrane potential $\left(\Delta \Psi_{\mathrm{m}}\right) .{ }^{4}$ This was a specific effect in the sense that the drop in of $\mathrm{DiOC}_{6}{ }^{3}$ incorporation was restricted to the SEB-reactive T-cell subset, which expresses the T-cell receptor variable chain $\beta 8$. Moreover, it could be truly shown in vivo, by labelling $T$ lymphocytes with intravenously injected $\Delta \Psi_{\mathrm{m}}$-sensitive dies. So we had identified a molecular marker, $\Delta \Psi_{\mathrm{m}}$ dissipation, that defined the subpopulation of $\mathrm{T}$ cells that would be eliminated in vivo in response to bacterial superantigens, glucocorticoids, ${ }^{4}$ self-antigens ${ }^{5}$ or infection with HIV-1. ${ }^{6}$ We soon realized that the loss of the $\Delta \Psi_{\mathrm{m}}$ marked an irreversible early step of the apoptotic process, observable in very different cell types, including epithelial cell lines and neurons, that it was controlled by Bcl-2-like proteins, ${ }^{4,7}$ that it mostly occurred before caspases were activated, and that it involved, at least in some cases, permeability transition, that is a process inhibited by ligands of the adenine nucleotide translocase and the mitochondrial cyclophilin D. ${ }^{8,9}$ However, we only became fully convinced that mitochondria would be part of the 'central executioner', when we constructed, back in 1995, a cell-free system in which we confronted purified mitochondria with purified nuclei. Upon induction of permeability transition, we found that mitochondria released an 'apoptosis-inducing factor' (that we retrospectively know to be a mixture of several proteins) that caused chromatin condensation and fragmentation when added to purified nuclei. $^{10}$ The mitochondrial release of these apoptogenic factors but not their action on the nucleus was found to be controlled by $\mathrm{Bcl}-2 .{ }^{11}$ Based on these observations, we constructed a general hypothesis that mitochondria would 'decide' the fate of cells and serve as a central integrator and coordinator of the apoptotic process. ${ }^{12}$

\section{CDD: What other early work was being done at the time on mitochondria?}

Martin Raff ${ }^{13}$ and Peter Krammer ${ }^{14}$ showed in 1994 that the nucleus was not required for apoptosis of human cells, and that a cytoplasmic regulator must exist. Donald Newmeyer ${ }^{15}$ et al. published at the end of 1994 that 'heavy membrane fractions', hesitantly called 'an organelle fraction enriched in mitochondria', can be required for apoptosis to occur is Xenopus egg extracts. Moreover, Bernard Mignotte et al. had found that SV40-transformed cell lines could reduce mitochondrial biogenesis and the $\Delta \Psi_{\mathrm{m}}$ upon apoptosis induction in vitro. $^{16}$ Patrice Petit, Bernard Mignotte and Marie-Lise Gougeon also reported that glucocorticoid-treated thymocytes manifested an early $\Delta \Psi_{\mathrm{m}}$ loss. ${ }^{17}$ However, the real confirmation of our claim that mitochondria would control apoptosis in mammalian cells came from Xiadong Wang's seminal discovery that cytochrome $c$ would be required for caspase activation in HeLa cell extracts. ${ }^{18}$ This latter finding was very much a relief for us. We had been standing alone in saying that mitochondria would be important for the regulation of apoptosis, and, at meetings, my talks only provoked glacial indifference and incredulous whispering. Even early in 1997, when I had given a lecture at a Keystone conference, one of my highly respected colleagues (he still is) stood up after my last slide, launching that 'mitochondria are bull shit' (sic). So, when Xiadong Wang published that cytochrome and the other proteins of the apoptosome-mediated caspase activation, ${ }^{18-}$ 20 and when several groups, with Xiadong Wang, Donald Newmeyer and Douglas Green as protagonists, confirmed that Bcl-2 stabilized mitochondrial membranes, ${ }^{21,22}$ the hostility that we had been experiencing progressively vanished. Without this help, we would have been forced to abandon our work.

\section{CDD: So the cytochrome $c$ is crucial, but what about other mitochondrial proteins?}

As I said, cytochrome $c$ turned out to be crucial for the activation of caspases via the intrinsic pathway. Xiaodong Wang identified cytochrome $c$ as an apoptogenic mitochondrial protein because his cell-free system was designed to detect caspase activation as the final readout. Instead, my group was looking for factors that would cause chromatin condensation, in the absence of cytosol, and thus we found AIF. Today, it is known that mitochondria can release dozens of different potentially apoptogenic proteins, with multiple distinct biochemical activities. ${ }^{23,24}$ Moreover, many different proteins regulate the permeability of mitochondrial membranes. In addition to the members of the Bcl-2 family, the first to be recognized to regulate mitochondrial permeability, ${ }^{25}$ 
proteins like $\mathrm{ANT},{ }^{26} \mathrm{VDAC}^{27}$ transcription factors such as p53, ${ }^{28}$ as well as lipid pores ${ }^{29}$ may contribute to the apoptotic permeabilization of mitochondrial membranes. So, it is probably wrong to concentrate on just one or a few proteins as being crucial regulators or mitochondrial apoptosis. Rather, it would be correct to regard mitochondrial membrane pemeabilization, on the whole, as a critical event in the death/life decision, beyond the molecular details. So my point is that we should, at least sometimes, concentrate on processes, rather than on individual molecules.

\section{CDD: What does AIF mean: 'artefact- inducing factor'?}

No, 'apoptosis-inducing factor'. But, I have to admit that I sometimes used the name that you insinuate in my lab to challenge the postdocs working on AIF. By the way, this is precisely the fear that I feel - and I suspect my colleagues as well - when we publish something really new. Do we report on a true, biologically relevant phenomenon or on an artefact (reproducible, though) without any meaning for real life? After we had published the discovery of AIF, in $1999,{ }^{30}$ and its knockout, together with Josef Penninger, in $2001,{ }^{31}$ confirmation of our data by independent groups was missing until 2002. It was only then that AIF was recognized to participate in $C$. elegans apoptosis ${ }^{32}$ and PARP-mediated mammalian cell death, ${ }^{33}$ just to give you a few examples.

\section{CDD: So how did you come across AIF?}

We identified and cloned AIF because we were looking for a protein that would be released from mitochondria and that would act on the nucleus, in a caspase-independent manner. AIF turned out to be a redox-active flavoproten, coded for by a nuclear gene, that is normally localised to the mitochondrial intermembrane space. ${ }^{30}$ Upon permeabilization of the outer membrane, AIF leaks out of mitochondria and translocates to the nucleus, where it interacts with DNA via positive charges clustered on its surface. ${ }^{34}$ One of the most fascinating aspects about AIF is that it is phylogenetically old. AIF exists in all eukaryotic kingdoms and even in archae- and eubacteria. AIF interacts with other evolutionary Methusalems such as cyclophilin A (which helps AIF to become a DNAse) ${ }^{35}$ and the heat shock protein HSP70 (which is the endogenous inhibitor of AIF). ${ }^{36}$ Moreover, as to be expected from a phylogenetically old protein, it intervenes in early ontogeny, as an obligate component of the molecular machinery that mediates the first wave of morphogenetic cell death during cavitation of the early embryo. ${ }^{31}$ We are right now working on the molecular details of the normal and apoptogenic functions of AIF.

\section{CDD: Does apoptosis influence cell metabolism?}

Of course, and vice versa. One of the intriguing aspects about apoptosis is that it is such a well orchestrated process that guarantees the rapid dismantling of virtually all cellular structures 'from inside', as well as 'from outside', by the lysosomal enzymes of adjacent cells that are stimulated to engulf the dying cell. Many investigators are educated in the belief that caspases are largely responsible for the perfect coordination of cellular catabolism. However, it is also possible that mitochondrial membrane permeabilization constitutes the explosive event occurring in a positive amplification loop, or if you prefer, a chain reaction, that liberates and activates dozens of different catabolic hydrolases, many of which are acting in a caspase-independent manner. It is difficult to imagine a more radical catabolic event than apoptosis, which, in addition, is tied to fulminant oxidation processes $^{7}$ and collapsing ion homeostasis. ${ }^{37}$ Again, these changes may well be coordinated by mitochondrial disruption.

\section{CDD: Were there any clinical implications of this work?}

A whole array of different implications. Let us just consider those pathologies involving an excess of cell death. The fact that mitochondria control apoptosis constitutes a therapeutic opportunity for the treatment of acute pathologies such as heart infarction and stroke, as well as, perhaps, chronic neurodegenerative diseases. Drugs are now being designed to prevent the inopportune permeabilization of mitochondrial membranes, by a direct action on mitochondria. ${ }^{38}$ My own laboratory has been focusing on the mechanisms how the human immunodeficiency virus (HIV-1) depletes cells from the immune system. We found that the HIV-1-encoded accessory protein $\mathrm{Vpr}$, a protein that can be secreted by HIV-1 infected cells and thus can act on uninfected cells, is actually capable of inducing apoptosis through permeabilizing interactions with mitochondrial proteins. ${ }^{39}$ The 'mitochondriotoxic' domain of Vpr required for this effect is frequently mutated in long-term nonprogressors, that is HIV-1 infected individuals who do not develop AIDS, ${ }^{40}$ underlining that Vpr is a virulence factor. In addition, we discovered a clinically relevant p53-dependent pathway stimulated by the HIV-1 envelope. Again, this lethal pathway involves an obligate mitochondrial permeabilization step. ${ }^{41}$

\section{CDD: If mitochondria controls immune interactions, how does it affect cancer? Why did you look on apoptosis and cancer?}

A cell death researcher may view cancer as the result of disabled apoptosis. This apoptosis resistance often involves a failure to transmit proapoptotic signals to mitochondria or is due to an enhanced resistance of mitochondria to permeabilization. As a result, it is tempting to conceive strategies of enforcing cell death by drugs that directly induce mitochondrial membrane permeabilization, for instance in a manner that is not inhibited by Bcl-2. Thus, we developed several procedures to overcome cancer-relevant apoptosis resistance, either by acting on mitochondrial proteins ${ }^{42,43}$ or by creating antagonists of the AIF inhibitor HSP70. ${ }^{44}$ I am aware of the fact that many pharmaceutical laboratories are in the process of attempting similar approaches. 


\section{CDD: Will you study other aspects of apoptosis, beyond the implication of mitochondria?}

Most researchers tend to follow up their discoveries and to continue within their area of interest, for instance by studying additional details or clinical applications. Since I am a more expansive type, I will deviate part of my efforts to tackle novel problems. I am very much interested in the question how cancer cell death might be manipulated to become immunogenic. ${ }^{45}$ Moreover, I have recently become intrigued by the possible interrelation between apoptosis and genomic instability. We have constructed an in vitro model in which simultaneous invalidation of cell cycle checkpoints and apoptosis can lead to chromosomal instability, ${ }^{46}$ and we are trying to show that this process may be relevant to early carcinogenesis... .

\section{CDD: So what happened?}

Well, exploring these new terrae incognitae I feel the same degree of excitement (and pleasure) as when we were working on mitochondria, 10 years ago. Not surprisingly, our new discoveries, in novel areas, are fulminantly rejected by the Editors of some major journals, exactly as this was the case when we submitted our first reports on mitochondrial apoptosis control. So, we must be on the right track.

\section{CDD: What music do you like to hear?}

I am quite flexible. I like Italian Opera, Spanish protest singers, American Jazz.... and since I am just back from a trip to Cuba, I am much under the Latin sway.

\section{CDD: Now tell us more on your reading}

I read a newspaper near-to-daily, in one of the five languages that I had the privilege to learn. My (last) favourite novelists are José Saramago, Antonio Tabucchi, Manuel Vazquez Montalban and Antonio Sciascia. And right now, I am reading the 'Genome war' by James Shreeve, a novel about the antagonism between private, commercial and public, scientific interest. I have the impression that we stand at a watershed, right now, in which our societies have to decide between economical jungle and civilized solidarity. And Science is at stake as well. Culture results from the juxtaposition of art and science, both of which are annihilated when they are reduced to mere merchandises.

\section{Acknowledgements}

Guido Kroemer is grateful to the funding bodies that supported his work, in particular ARC, ANRS, CNRS, EU, FRM, INSERM, Ligue contre le
Cancer, Ministry for Science, and Sidaction. He is indebted to many colleagues who contributed help, reagents, ideas and discussions, but Guido Kroemer would especially like to acknowledge the enlightenment by and friendship of Catherine Brenner, Carmen Garrido, John C Reed, Josef Penninger, Mauro Piacentini, Laurence Zitvogel, as well as of the current members of his laboratory. Special thanks to Maria Castedo, Didier Decaudin, Antonio Macho, Philippe Marchetti, Patrice Petit, Santos A Susin and Naoufal Zamzami for contributing to the early steps of the 'mitochondrial revolution'.

1. Wyllie AH (1980) Nature 284: $555-556$

2. Gonzalo JA et al. (1992) Eur. J. Immunol. 22: 1007-1011

3. Gonzalo JA et al. (1993) J. Exp. Med. 177: 1239-1246

4. Zamzami N et al. (1995) J. Exp. Med. 181: 1661-1672

5. Castedo M et al. (1995) Eur. J. Immunol. 25: 3277-3284

6. Macho A et al. (1995) Blood 86: 2481-2487

7. Zamzami N et al. (1995) J. Exp. Med. 182: 367-377

8. Zamzami $\mathrm{N}$ et al. (1996) FEBS Lett. 384: 53-57

9. Marchetti $P$ et al. (1995) J. Exp. Med. 184: 1155-1160

10. Zamzami $\mathrm{N}$ et al. (1996) J. Exp. Med. 183: 1533-1544

11. Susin SA et al. (1996) J. Exp. Med. 184: 1331-1342

12. Kroemer $\mathrm{G}$ et al. (1995) FASEB J. 9: 1277-1287

13. Jacobson MD et al. (1994) EMBO J. 13: 1899-1910

14. Schulze-Osthoff K et al. (1994) J. Cell Biol. 127: 15-20

15. Newmeyer DD, Farschon DM and Reed JC (1994) Cell 79: 353-364

16. Vayssière J-L et al. (1994) Proc. Natl. Acad. Sci. USA 91: 11752-11756

17. Petit PX et al. (1995) J. Cell Biol. 130: 157-167

18. Liu XS et al. (1996) Cell 86: 147-157

19. Zhou H et al. (1997) Cell 90: 405-413

20. Li P et al. (1997) Cell 91: 479-489

21. Yang J et al. (1997) Science 275: 1129-1132

22. Kluck RM et al. (1997) Science 275: 1132-1136

23. Patterson S et al. (2000) Cell Death Differ. 7: 137-144

24. Van Loo G et al. (2002) Cell Death Differ. 9: 301-308

25. Kroemer G. (1997) Nat. Med. 3: 614-620

26. Marzo I et al. (1998) Science 281: 2027-2031

27. Shimizu S et al. (1999) Nature 399: 483-487

28. Marchenko ND et al. (2000) J. Biol. Chem. 275: 16202-16212

29. Kuwana T et al. (2002) Cell 111: 1-12

30. Susin SA et al. (1999) Nature 1397: 441-446

31. Joza $\mathrm{N}$ et al. (2001) Nature 20410: 549-554

32. Wang $X$ et al. (2002) Science 298: 1587-1592

33. Yu SW et al. (2002) Science 297: 259-263

34. Ye $\mathrm{H}$ et al. (2002) Nat. Struct. Biol. 9: 680-684

35. Cande C et al. (2004) Oncogene 23: 1514-1521

36. Gurbuxani $S$ et al. (2003) Oncogene 22: 6669-6678

37. Dallaporta B et al. (1998) J. Immunol. 160: 5605-5615

38. Mattson MP and Kroemer G (2003) Trends Mol. Med. 9: 196-205

39. Jacotot $E$ et al. (2001) J. Exp. Med. 193: 509-520

40. Brenner $C$ and Kroemer G (2003) J. Clin. Invest. 111: 1455-1457

41. Perfettini J-L et al. (2004) J. Exp. Med. 199: 629-640

42. Decaudin D et al. (2002) Cancer Res. 62: 1388-1393

43. Debatin KM, Poncet D and Kroemer G (2002) Oncogene 21: 8786-8803

44. Schmitt E (2003) Cancer Res. 63: 8233-8240

45. Zitvogel $L$ (2004) Adv. Immunol., in press

46. Castedo M (2004) Oncogene 23: 2825-2837 